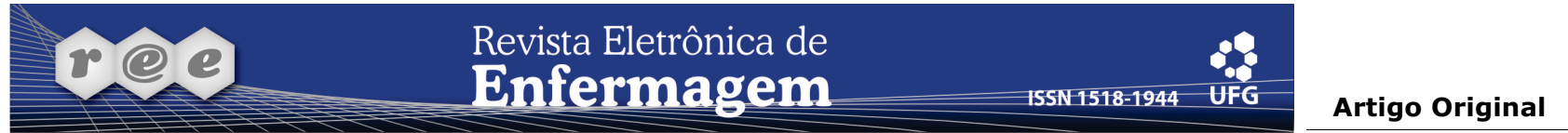

\title{
Análise do fluxo de atendimento de serviço hospitalar de emergência: estudo de caso
}

Analysis of the emergency hospital patient flow: a case study

Análisis del flujo de atención de servicio de urgencia en hospital: estudio de caso

José Aparecido Bellucci Júnior ${ }^{1}$, Laura Misue Matsuda², Sonia Silva Marcon ${ }^{3}$

${ }^{1}$ Enfermeiro, Mestre em Enfermagem. Professor Auxiliar da Universidade Estadual do Norte do Paraná. Bandeirantes, PR, Brasil. E-mail:

bellucci@uenp.edu.br.

${ }^{2}$ Enfermeira, Doutora em Enfermagem Fundamental. Professora Adjunta da Universidade Estadual de Maringá (UEM). Maringá, PR, Brasil. E-mail:

Imisue@terra.com.br.

${ }^{3}$ Enfermeira, Doutora em Filosofia da Enfermagem. Professora Associada da UEM. Maringá, PR, Brasil. E-mail: ssmarcon@uem.br.

\section{RESUMO}

Estudo qualitativo, modalidade Estudo de Caso que objetivou analisar o fluxo de atendimento em dois Serviços Hospitalares de Emergência. O estudo foi realizado entre novembro e dezembro de 2010, em um Serviço que atende por meio do Acolhimento com Classificação de Risco e, em outro, por ordem de chegada. Para a coleta de dados, utilizouse como técnica a observação direta da prática, com registros em notas de campo que foram tratados com o uso da técnica análise de conteúdo. Das análises das informações coletadas, resultaram duas categorias temáticas: Cuidando do usuário em Serviços Hospitalares de Emergência e Acolhendo o usuário e familiares. Conclui-se que, nos dois Serviços investigados, não há sistema de contrarreferenciamento; o atendimento no Serviço que adota o Acolhimento com Classificação de Risco é contínuo e hierarquizado; o fluxo de pacientes é mais dinâmico, e o enfermeiro tem mais autonomia na tomada de decisão clínica.

Descritores: Enfermagem; Serviço Hospitalar de Emergência; Acolhimento; Enfermagem em Emergência; Triagem.

\section{ABSTRACT}

This qualitative study, using a case study design, had the aim to analyze the service flow at two emergency hospital units. The study was developed in November and December 2010, at a service where care is provided by means of embracement with risk classification and at another one where patients are seen on a first come, first served basis. Data were collected by means of direct observation of the practice, with records made on field notes that were treated with the content analysis technique. The analyses of the collected information resulted in two thematic categories: Caring for users at emergency hospital services and Embracing users and families. In conclusion, neither of the services had a counter-referral system; care at the service that adopts embracement with risk classification was continuous and hierarchized; the flow of patients was more dynamic and; the nurse had more autonomy for making clinical decisions.

Descriptors: Nursing; Emergency Service, Hospital; User Embracement; Emergency Nursing; Triage.

\section{RESUMEN}

Estudio cualitativo, modalidad Estudio de Caso, objetivando analizar el flujo de atención en dos Servicios de Urgencias en Hospital. Estudio realizado entre noviembre y diciembre de 2010, en un Servicio que atiende por Acogimiento por Clasificación de Riesgo; en otro, por orden de llegada. Los datos fueron recolectados a través de la observación directa de la práctica, con registros en notas de campo, tratados con la técnica del análisis de contenido. Del análisis resultaron dos categorías temáticas: Cuidando del Usuario en Servicios de Urgencia en Hospital y Acogiendo al Usuario y Familiares. Se concluye en que en los dos servicios estudiados existe un sistema contra referencial; la atención den el Servicio que adoptó el Acogimiento con Clasificación de Riesgo es continuo y jerarquizado; el flujo de pacientes es más dinámico; y el enfermero posee mayor autonomía en la toma de decisiones clínicas.

Descriptores: Enfermería; Servicio de Urgencia en Hospital; Acogimiento; Enfermería de Urgencia; Triaje. 


\section{INTRODUÇÃO}

Os Serviços Hospitalares de Emergências (SHE), ou Prontos Socorros, são unidades complexas que devem possuir apenas leitos de observação, destinados ao atendimento de usuários com ou sem risco de morte, cujos agravos necessitam de intervenções imediatas nas 24 horas do $\mathrm{dia}^{(1)}$.

Apesar de o SHE ser considerado como a principal porta de entrada no sistema de saúde brasileiro, a baixa resolubilidade e a elevada procura pelo Serviço dificultam a obtenção de resultados que sejam positivos em relação ao atendimento prestado ${ }^{(2)}$, pois o tempo gasto com as consultas de baixa complexidade e o excesso de usuários que lá retornam, interferem negativamente na agilidade e na segurança dos procedimentos executados.

Ao pensar em qualidade do atendimento em SHE, torna-se necessária a definição dos fluxos para o atendimento ao usuário, de modo que contemple aspectos peculiares dos processos de trabalho e acesso do usuário, com vistas ao melhor aperfeiçoamento e efetividade da atenção $^{(3)}$.

A escassez de alternativas para o acesso ao atendimento contribui para que a maioria das pessoas idealize o SHE como a única opção para resolver todo e qualquer problema de saúde. Para minimizar esse quadro, os gestores hospitalares têm se utilizado de diversas estratégias relacionadas ao elevado fluxo de atendimento, como: implantação da unidade de pronto-atendimento; inserção do sistema de gerência de fluxo de pacientes do SHE para os leitos de internação; aumento do número de integrantes da equipe de enfermagem; ampliação de leitos da unidade; entre outras ${ }^{(4)}$.

Em relação ao controle de fluxo de entrada e saída de pacientes em $\mathrm{SHE}$, uma das estratégias mais utilizadas é a organização do atendimento por meio da utilização de protocolos de classificação do risco para priorizar os casos mais graves ${ }^{(5)}$. Nesses protocolos, a classificação do grau de urgência é realizada pelo enfermeiro que avalia rapidamente o caso a partir da reunião de informações contidas no histórico, no exame físico e nas queixas do paciente ${ }^{(6)}$.

No contexto mundial, a utilização de protocolos de classificação de risco em SHE teve início em meados de 1970, na Austrália. Em 1990, foi impulsionado pela informatização nos hospitais e; atualmente, é utilizado como critério para avaliação nas unidades de emergência ${ }^{(7)}$.

Nos Estados Unidos da América e no Canadá, a maior parte dos Serviços de Emergência se utiliza dos sistemas Emergency Severity Index (ESI) e Canadian Emergency
Department Triage and Acuity Scale (CTAS) para classificar o grau de prioridade do usuário para o atendimento $^{(8-9)}$. Esses dois sistemas de classificação permitem que a atenção ao usuário ocorra de acordo com cinco níveis de complexidade, e relacionam desde Non Urgent (Nivel 5) a Ressuscitation (Nivel 1).

Na Suécia, país onde mais de $97 \%$ dos atendimentos em SHE ocorrem por meio de sistemas de Classificação de Risco, há relatos de que houve melhoria no fluxo de pacientes e maior agilidade na condução diagnóstica dos $\operatorname{casos}^{(10)}$. Por outro lado, na Holanda, mais de $31 \%$ dos SHE não se utilizam de nenhum tipo de sistema de Classificação de Risco e isso, tem resultado em fluxos de atendimentos pouco ordenado e não hierarquizado de acordo com as prioridades para o atendimento ${ }^{(3)}$.

No Brasil, o Ministério da Saúde (MS), por meio da Política Nacional de Humanização, propôs em 2004, a diretriz Acolhimento com Classificação de Risco (ACCR) ${ }^{(11)}$, a qual é considerada como uma das principais estratégias para a promoção da qualidade no atendimento em SHE, pois além de acolher e classificar o usuário de acordo com a gravidade de cada caso, possibilita a construção e a pactuação de redes e fluxos com as Unidades Básicas de Saúde, que de acordo com os propósitos do Sistema Único de Saúde brasileiro, devem funcionar como porta de entrada para a grande maioria dos usuários dos serviços de saúde ${ }^{(12)}$. Nessa perspectiva, o funcionamento do ACCR ocorre por meio da recepção do usuário no $\mathrm{SHE}$, encaminhamento à Consulta de Enfermagem e atendimento médico, segundo o grau de urgência.

Dado ao crescente número de pacientes em SHE, destaca-se a importância do enfermeiro na administração e gerenciamento de fluxo nesses serviços ${ }^{(13)}$. Sob esta ótica, estudos que analisam o fluxo de usuários em SHE que adotam sistemas diferentes de atendimento ao público são importantes e necessários porque, além de produzir evidências científicas sobre o impacto do ACCR no atendimento, pode induzir os trabalhadores a refletirem sobre o processo de trabalho e à identificação de aspectos nos quais se concentram os principais problemas.

No intuito de verificar aspectos relacionados ao ACCR em SHE, pergunta-se: Como se processa o fluxo de atendimento aos usuários em SHE? Para responder a essa questão, este estudo tem como objetivo analisar o fluxo de atendimento ao usuário em dois SHE com modalidades/sistemas diferentes de atendimento. 


\section{CAMINHO METODOLÓGICO}

Estudo qualitativo, modalidade Estudo de Caso, que fez uso da observação direta da prática como técnica de coleta de dados, a qual consiste na observação e registro de comportamentos, sem a participação dos observados $^{(14)}$.

A observação foi do tipo conhecida pelos observados, ou seja, quando havia interesse os profissionais eram informados sobre o objetivo do estudo e a função do observador, o qual se apresentava no serviço, de jaleco branco e com crachá que o identificava.

O estudo foi realizado no SHE de dois Hospitais, denominados SHE I e SHE II.

O SHE I é um Hospital Filantrópico, de Atendimento Geral, do interior do Estado de São Paulo, existe desde 1943 e atende cerca de 9000 pacientes/mês, possui 144 leitos, com média de 10.470 consultas/mês. Atualmente possui 12 salas para atendimento; 25 leitos efetivos; tem porta aberta durante as 24 horas do dia e é referência para atendimento emergencial para 27 cidades da região. Possui o ACCR implantado desde março de 2008 o qual funciona durante o período das $07 \mathrm{~h} 00$ às $01 \mathrm{~h} 00$. Todos os usuários que procuram o Serviço no período de funcionamento do ACCR são avaliados por meio da Consulta de Enfermagem.

O SHE II é um Hospital de Ensino, de Administração Indireta - Autarquia, de Atendimento Geral, do interior do Estado do Paraná, existe desde 1989 e atende em média 4.000 pacientes/mês, possui 31 leitos efetivos, porém acomoda em média 90 pacientes/dia, tem porta aberta durante as 24 horas do dia, é referência para atendimento na área de traumatologia e alta complexidade e oferece atendimento nas principais especialidades.

As sessões de observações, que tiveram duração média de duas horas, foram realizadas em 2010, no período de 01 a 20 de novembro no SHE I e de 22 de novembro a 12 de dezembro no SHE II, em diferentes turnos e momentos, inclusive nos finais de semana.

As informações registradas compreenderam as ações, comportamentos, gestos, atitudes e palavras que visaram identificar as formas como ocorrem os fluxos de atendimento com relação a: realização de exames diagnósticos; atendimento médico e de enfermagem; internação e alta; informações aos familiares e; atendimento na Recepção.

A partir do material coletado, foi realizado o tratamento dos dados por meio da técnica Análise de Conteúdo, modalidade Temática, desenvolvida em três etapas: Pré-exploração do material, Seleção das unidades de análise e Categorização. Em seguida, as unidades temáticas foram revisadas para apreensão das categorias empíricas, correspondentes ao processo de atendimento.

Das informações coletadas emergiram as seguintes categorias: "Cuidando do usuário no SHE" e "Acolhendo o usuário e familiares/acompanhantes". Em cada categoria, os dados foram agregados na forma de subcategorias temáticas e itens observados, os quais significam respectivamente, os diferentes fluxos que ocorrem no processo de atendimento e ações de trabalho de cada Serviço.

Este estudo foi subsidiado pela Fundação Araucária Apoio ao Desenvolvimento Científico e Tecnológico do Paraná e o Projeto de Pesquisa foi aprovado pelo Comitê Permanente de Ética em Pesquisas com Seres Humanos da Universidade Estadual de Maringá, sob o parecer no $606 / 2010$.

\section{RESULTADOS}

A exploração das observações registradas sobre o Processo de Atendimento resultou em duas categorias e seis subcategorias temáticas.

\section{Cuidando do usuário no SHE}

As principais observações agrupadas nesta categoria estão descritas no Quadro 1 e se referem ao fluxo do atendimento ao usuário nos dois $\mathrm{SHE}$, relacionado à: realização de exames diagnósticos; atendimento médico e de enfermagem e; internação/alta.

\section{Acolhendo o usuário e familiares/acompanhantes.}

As principais observações agrupadas nesta categoria se relacionam aos encaminhamentos dos pacientes após o fornecimento de informações sobre o estado de saúde deste a familiares/acompanhantes e também, sobre o fluxo do atendimento no Serviço de Recepção. 
Quadro 1: Fluxos e procedimentos do processo de atendimento. Maringá, PR, Brasil, 2010.

\begin{tabular}{|c|c|c|c|}
\hline \multirow{2}{*}{ Subcategorias } & \multirow{2}{*}{$\begin{array}{c}\text { Itens } \\
\text { observados }\end{array}$} & \multicolumn{2}{|c|}{ Instituições } \\
\hline & & SHE I & SHE II \\
\hline \multirow{2}{*}{$\begin{array}{l}\text { Fluxo interno para } \\
\text { realização de exames } \\
\text { diagnósticos }\end{array}$} & $\begin{array}{c}\text { Exames } \\
\text { Laboratoriais }\end{array}$ & \multirow{2}{*}{$\begin{array}{l}\text { Solicitação informatizada; prioridade na } \\
\text { realização e devolução segundo a } \\
\text { classificação do ACCR. }\end{array}$} & \multirow{2}{*}{$\begin{array}{c}\text { Solicitação manual em impresso } \\
\text { com duas vias, identificado com a } \\
\text { palavra EMERGÊNCIA quando } \\
\text { necessário. }\end{array}$} \\
\hline & $\begin{array}{l}\text { Exames de } \\
\text { Imagem }\end{array}$ & & \\
\hline \multirow{2}{*}{$\begin{array}{l}\text { Fluxo do usuário para } \\
\text { atendimento médico }\end{array}$} & Local de espera & $\begin{array}{l}\text { O usuário aguarda pelo atendimento } \\
\text { médico em local específico, de acordo } \\
\text { com a classificação do seu agravo. }\end{array}$ & $\begin{array}{c}\text { Todos os usuários são destinados a } \\
\text { um único local, sem distinção de } \\
\text { prioridade para o atendimento. }\end{array}$ \\
\hline & Consulta médica & $\begin{array}{l}\text { O usuário é chamado de acordo com a } \\
\text { Classificação do ACCR na seguinte } \\
\text { ordem: Amarelo, Verde e Azul. }\end{array}$ & $\begin{array}{c}\text { O usuário é chamado para o } \\
\text { atendimento médico de acordo com } \\
\text { a ordem de chegada. }\end{array}$ \\
\hline \multirow{3}{*}{$\begin{array}{l}\text { Fluxo do usuário para o } \\
\text { atendimento de } \\
\text { enfermagem }\end{array}$} & $\begin{array}{l}\text { Consulta de } \\
\text { Enfermagem }\end{array}$ & $\begin{array}{l}\text { Após abertura da Ficha de Atendimento } \\
\text { Ambulatorial (FAA) o usuário é } \\
\text { encaminhado à Consulta de Enfermagem. }\end{array}$ & $\begin{array}{l}\text { Não existe Consulta de } \\
\text { Enfermagem ao usuário. }\end{array}$ \\
\hline & $\begin{array}{l}\text { Anotações de } \\
\text { Enfermagem }\end{array}$ & $\begin{array}{l}\text { São realizadas nas } F A A \text {, desde a } \\
\text { recepção até a alta. }\end{array}$ & $\begin{array}{c}\text { Só existem para os usuários } \\
\text { internados ou às intercorrências } \\
\text { surgidas durante a permanência no } \\
\text { setor. }\end{array}$ \\
\hline & $\begin{array}{l}\text { Procedimentos de } \\
\text { enfermagem }\end{array}$ & $\begin{array}{l}\text { Após a consulta médica o usuário que } \\
\text { necessita ser medicado é conduzido ao } \\
\text { Posto de Enfermagem. }\end{array}$ & Idem \\
\hline \multirow{3}{*}{$\begin{array}{l}\text { Fluxo do usuário à } \\
\text { internação/alta }\end{array}$} & $\begin{array}{l}\text { Tempo de } \\
\text { permanência }\end{array}$ & $\begin{array}{l}\text { É regulado por uma Central de Vagas } \\
\text { interna. Após } 24 \mathrm{~h} \text { o usuário não } \\
\text { permanece mais no SHE. Dispõe de leitos } \\
\text { temporários de internação. }\end{array}$ & $\begin{array}{l}\text { Pode variar desde um breve } \\
\text { atendimento até semanas de } \\
\text { internação. Dispõe de leitos fixos } \\
\text { para internação. }\end{array}$ \\
\hline & Internação & $\begin{array}{l}\text { A Central de Vagas Interna localiza um } \\
\text { leito, comunica a enfermagem e efetua a } \\
\text { internação. }\end{array}$ & $\begin{array}{l}\text { O usuário aguarda vaga para } \\
\text { internação na enfermaria, no } \\
\text { corredor ou na Sala de } \\
\text { Observações. }\end{array}$ \\
\hline & Alta & $\begin{array}{l}\text { As altas de internações a partir do SHE } \\
\text { são raras; não existe um sistema de } \\
\text { orientação para altas das observações. }\end{array}$ & $\begin{array}{c}\text { As altas de internações no SHE são } \\
\text { frequentes; não existe um sistema } \\
\text { de orientação nas altas das } \\
\text { observações. }\end{array}$ \\
\hline
\end{tabular}

Quadro 2: Ações de Acolhimento ao usuário e familiares. Maringá, PR, Brasil, 2010.

\begin{tabular}{|c|c|c|c|}
\hline \multirow{2}{*}{ Subcategorias } & \multirow{2}{*}{ Itens } & \multicolumn{2}{|c|}{ Instituições } \\
\hline & & SHE I & SHE II \\
\hline \multirow[t]{2}{*}{$\begin{array}{l}\text { Fluxo de } \\
\text { informações aos } \\
\text { familiares/ } \\
\text { acompanhantes }\end{array}$} & Boletim Informativo & $\begin{array}{l}\text { A Cada troca de plantão é } \\
\text { disponibilizado ao Serviço Social um } \\
\text { Boletim com informações sobre o } \\
\text { estado de saúde dos usuários. }\end{array}$ & $\begin{array}{l}\text { Não existe. Quando solicitado, o } \\
\text { médico de plantão fornece } \\
\text { informações ao paciente e familiares. } \\
\text { As informações são fornecidas pela } \\
\text { enfermagem e, quando presentes, } \\
\text { pelos médicos residentes. }\end{array}$ \\
\hline & Visitas de familiares & $\begin{array}{c}\text { Existem dois horários específicos para a } \\
\text { visita de familiares e troca de } \\
\text { acompanhantes. }\end{array}$ & $\begin{array}{l}\text { Não foi observado horário fixo para } \\
\text { visita de familiares. }\end{array}$ \\
\hline \multirow[b]{2}{*}{$\begin{array}{l}\text { Fluxo de usuários } \\
\text { para o atendimento } \\
\text { na Recepção }\end{array}$} & $\begin{array}{l}\text { Recepção e } \\
\text { orientações ao } \\
\text { usuário }\end{array}$ & $\begin{array}{c}\text { O usuário é acolhido por um } \\
\text { colaborador que orienta as dúvidas e } \\
\text { direciona o fluxo para a abertura da } \\
\text { FAA. }\end{array}$ & $\begin{array}{l}\text { O Serviço de Recepção é a referência } \\
\text { para obtenção de informações } \\
\text { relacionadas somente à localização do } \\
\text { cliente no setor. }\end{array}$ \\
\hline & $\begin{array}{l}\text { Preenchimento e } \\
\text { encaminhamento da } \\
\text { FAA }\end{array}$ & $\begin{array}{l}\text { Mediante o preenchimento da FAA pelo } \\
\text { Serviço de Recepção, o usuário é } \\
\text { direcionado à Consulta de Enfermagem } \\
\text { que, após ser avaliado, é conduzido a } \\
\text { uma das salas correspondentes a sua } \\
\text { classificação. }\end{array}$ & $\begin{array}{l}\text { Após o preenchimento da FAA pelo } \\
\text { Serviço de Recepção o usuário é } \\
\text { direcionado a um lugar comum para } \\
\text { esperar pelo atendimento, de acordo } \\
\text { com a especialidade e a ordem de } \\
\text { chegada. }\end{array}$ \\
\hline
\end{tabular}

\section{DISCUSSÃO}

\section{Cuidando do usuário no SHE}

A qualidade do cuidado em SHE é influenciada pela organização do serviço e também pela criação de vínculo entre o usuário e o trabalhador. Nesse sentido, quando se discute a estrutura para dar suporte ao atendimento nesse Serviço, a análise criteriosa do fluxo de realização de exames diagnósticos, atendimento médico, de enfermagem e internação/alta hospitalar são fundamentais para a organização das ações da equipe de saúde. 
Fluxo interno para realização de exames diagnósticos

As observações registradas nesta subcategoria refletem como se apresenta a organização do fluxo de envio, análise e devolução dos resultados de exames diagnósticos. Nesse caso, a análise do fluxo é importante porque o tempo de espera do usuário para obter os resultados de exames pode ser um dos fatores que congestionam o atendimento.

No SHE II, por exemplo, os resultados dos exames laboratoriais demoram cerca de três horas e os exames de imagem, apesar de serem liberados logo após a realização, não são acompanhados pelos laudos. Muitas vezes, por falta de comunicação da equipe médica com a equipe de enfermagem, à solicitação de exame (laboratório ou RX) é depositada na pasta do prontuário e só é descoberto no final do turno de trabalho, quando os profissionais de enfermagem realizam a checagem dos medicamentos.

As observações referentes aos dois itens dessa subcategoria revelam que o SHE I é mais ágil do que o SHE II na notificação das solicitações e nos resultados dos exames diagnósticos porque, aquele, possui sistema informatizado que além de organizar os pedidos, relaciona o grau de urgência do exame, de acordo com a classificação atribuída ao usuário pelo ACCR.

Em consonância com esses achados, um estudo europeu constatou que nos SHE em que há um enfermeiro classificando o grau de urgência do atendimento, por meio de um sistema informatizado, o tempo de realização do diagnóstico e tratamento da doença foi reduzido. Cabe salientar ainda que, de acordo com esse estudo, alguns protocolos locais permitem ao "enfermeiro de triagem" o diagnóstico e tratamento de acometimentos considerados lesões menores como: pequenas fraturas; objetos estranhos na pele; infecções de olhos, ouvido e garganta, entre outros ${ }^{(15)}$.

Percebe-se que os benefícios da informatização no SHE se estendem além da rapidez no processamento da informação e devolução dos resultados porque, o seu uso diminui extravios de pedidos impressos; prioriza o atendimento às urgências e emergências; disponibiliza dados diversos como o histórico do usuário para a consulta rápida e; minimiza erros de solicitações ${ }^{(16)}$. Destarte, em serviços hospitalares em que o tempo é fator determinante à sobrevida do paciente, o trabalho informatizado, além de proporcionar maior segurança à equipe e agilidade na condução clínica, também constitui ferramenta importante à Sistematização da Assistência de Enfermagem por garantir acesso rápido às informações.
De acordo com informações não formais obtidas no SHE I, antes da implantação do ACCR a solicitação de exames já era informatizada, mas os exames não eram realizados de acordo com o grau de urgência, o que, em parte, retardava o atendimento. Por ocasião da coleta de dados, como a realização de exames é organizada segundo o critério de risco do usuário, percebeu-se que os setores de apoio do SHE I têm relação direta e imediata com a resolubilidade do Serviço de Urgência e isso, é estabelecido no Documento Básico do Ministério da Saúde, o qual orienta a sistematização do $\mathrm{ACCR}^{(12)}$.

No SHE II, apesar de a solicitação de exames ser realizada manualmente, quando especificado como "urgência/emergência" os exames são realizados com maior rapidez e os resultados são disponibilizados em cerca de 30 minutos.

\section{Fluxo do usuário para o atendimento médico}

Essa subcategoria apresenta dados referentes a um dos momentos mais críticos do processo de atendimento, qual seja: a espera pela consulta médica.

No SHE I existem locais específicos para o usuário aguardar pelo atendimento médico, de acordo com a prioridade do agravo.

No SHE II a realidade se assemelha a da maioria dos SHE do Brasil, que não classifica o risco do usuário, pois os pacientes aguardam pelo atendimento médico em um único local, conforme a ordem de chegada. Nesse Serviço, os funcionários da Recepção são responsáveis pelo preenchimento inicial da FAA e determinam a prioridade do atendimento com base nos dados e impressões referidos pelo usuário. Assim, caso haja percepção de urgência, o próprio funcionário é que se encarrega de comunicar a equipe médica e/ou de enfermagem.

Na forma de atendimento do SHE II, se observa que somente os casos emergenciais, se identificados à primeira vista ou quando são conduzidos pelo serviço de atendimento pré-hospitalar, são atendidos com prioridade. Isso é preocupante porque, nesses casos, pode ocorrer subestimação da dor dos pacientes que aguardam pela consulta, o que compromete a avaliação segura do real estado clínico do indivíduo ${ }^{(17)}$.

Em relação ao trabalho médico no SHE I, segundo relatos dos trabalhadores, após dois anos de implantação do ACCR, os profissionais que antes resistiam em atuar conforme esse modelo de atendimento parecem ter se integrado à modalidade de trabalho em equipe, pois referem que o sistema tornou $o$ atendimento mais organizado, humano e acolhedor. 
No SHE II, a rotina médica é direcionada para atender os casos não referenciados e às emergências evidentes. Nesse aspecto, em meio ao elevado contingente de falsa demanda, é eminente o perigo de se ter casos graves não identificados porque, usuários que poderiam ter seus problemas de saúde resolvidos em outros níveis de assistência, mas que por comodismo, facilidade, ou até mesmo para faltar ao trabalho, procura atendimento nos SHE.

Consta que em alguns locais dos EUA, há médicos específicos no SHE para atender pacientes classificados pelo enfermeiro. Nessa forma de atuação, existem médicos especializados para atender os casos de urgência e emergência e outros, para atender às não urgências ${ }^{(18)}$, caracterizando um método inovador de gerir o fluxo do usuário para o atendimento médico.

\section{Fluxo do usuário para o atendimento de enfermagem}

Esta subcategoria reflete como é o fluxo do usuário para o atendimento de enfermagem nos serviços observados. Considera-se como "atendimento de enfermagem em SHE" os cuidados dispensados aos usuários desde a abordagem primária até a alta do setor, incluindo os registros pertinentes às atividades, intercorrências e administração de medicamentos prescritos.

Nessa subcategoria, devido à estreita ligação entre os três itens observados (Consulta de Enfermagem; Procedimentos de Enfermagem e Anotação de Enfermagem), a discussão dos mesmos ocorrerá de forma conjunta.

No SHE I, as atividades são dispensadas a todos os usuários, durante o horário de funcionamento do ACCR. Já no SHE II, apesar de os "Procedimentos de Enfermagem" ocorrer em quantidade elevada, a Consulta e as anotações de enfermagem não são realizadas na abordagem primária ao usuário.

Cabe ressaltar que durante a Consulta de Enfermagem no ACCR, o papel do enfermeiro ganha notoriedade porque, além de outros dados, também se registram as orientações fornecidas. Acresça-se a isso, o fato de que a aproximação do enfermeiro com o usuário, por meio da consulta de enfermagem, favorece a promoção da saúde devido à disseminação de informações referentes ao processo de educação em saúde ${ }^{(19)}$.

Em sendo assim, o fato de não existir Consulta e anotações de enfermagem na abordagem "de porta" ao usuário no SHE, configura como não conformidade na atuação do enfermeiro nesse Serviço e isso pode dificultar a realização de práticas seguras e acolhedoras no processo de cuidado. Diante desse quadro, pondera-se que, além de organizar o fluxo de atendimento, o ACCR melhora a documentação, a comunicabilidade das informações e com isso, a chance de resolver o problema do usuário.

Em que pese o fato de se observar que no SHE I os registros de enfermagem ocorrem com maior frequência, durante o período de funcionamento do ACCR (07:00 às 01:00h), de maneira geral, mesmo no período em que o ACCR não funciona, as informações referentes aos procedimentos de enfermagem são registradas de forma mais frequente e completa. Isso possivelmente ocorre porque no ACCR os trabalhadores se habituam a realizar anotações de forma rotineira, relacionadas às queixas e procedimentos. Por outro lado, quando a equipe de enfermagem não é preparada para atuar no ACCR, a avaliação padrão de entrada fica prejudicada, pois os registros realizados de forma inadequada e; às classificações imprecisas, tendem a prejudicar 0 tratamento e o fluxo de atendimento ${ }^{(20)}$.

Com relação às Anotações de Enfermagem, apesar de serem consideradas como meio de se garantir a qualidade da assistência prestada, se percebe que no SHE II esse procedimento é realizado somente em casos de internação ou quando ocorrem intercorrências com o usuário. Como implicação, evidencia-se morosidade ou até ausência de reavaliação médica em pacientes com potencial de agravamento do caso, o que certamente contraria um dos preceitos do dispositivo ACCR, o qual reza pela aproximação dos profissionais de saúde com os usuários atendidos no SHE. Em circunstância como a referida, no SHE II, pôde ser observada falta de organização no fluxo para o atendimento de enfermagem, principalmente no que se refere às Consultas e Anotações de Enfermagem.

\section{Fluxo do usuário à internação/alta}

O acúmulo de pacientes que aguardam por internação nos SHE é motivo de preocupação de gestores e equipe de saúde porque, nesses Serviços, a estrutura para atender o usuário deve ser organizada para o atendimento emergencial, estabilização e encaminhamento à alta ou à internação em unidade específica.

Problemas relacionados com a falta de leitos para internação ocorrem nos dois Serviços investigados, porém em virtude da complexidade dos casos atendidos no SHE II, o acúmulo de usuários internados é maior que no SHE I. Um fator que seguramente favorece este Serviço é a existência de uma Central Interna de Regulação de Leitos que controla o tempo de espera e direciona os usuários de 
acordo com a disponibilidade de vagas e a gravidade dos casos.

Com o intuito de melhorar o fluxo de pacientes destinados a internação em SHE, enfermeiros que atuam no Department of Emergency Medicine, York Hospital Estados Unidos da América, desenvolveram o Triage Nurse Prediction (TNP) que consiste numa ferramenta que objetiva reduzir o tempo de espera por leito de internação, que prevê, no momento da Classificação de Risco, se o paciente será internado. A aplicação desse instrumento é realizada por enfermeiros e consta que, no serviço em que foi utilizado, houve redução do tempo de espera por leito de internação ${ }^{(5)}$.

No Brasil, uma das estratégias atuais, utilizadas para dirimir à problemática antes referida é a implantação do trabalho em Redes de Atenção a Saúde (RAS), cujo modelo de atendimento se pauta na integração entre pontos de atenção (Unidades de Saúde) que se comunicam entre $\mathrm{si}^{(21)}$, para o direcionamento do usuário a um Serviço ou Unidade de Saúde e assim, ser atendido conforme o grau do seu agravo.

Especialmente ao SHE II, estudo realizado em 2002 constatou que apenas $10 \%$ dos casos de menor complexidade eram referenciados às Unidades Básicas de Saúde (UBS) ${ }^{(22)}$. Não obstante, passados mais de uma década, se observa ausência de um mecanismo de contrarreferência estruturado e isso, tem resultado em congestionamento "de porta".

\section{Acolhendo o usuário e familiares/acompanhantes}

$\mathrm{Na}$ perspectiva de que a definição pela escolha do serviço de saúde parte das experiências vividas pelos familiares e pelo próprio usuário, estudos sobre fluxos de informações a familiares e usuários, relacionadas ao atendimento da Recepção nos SHE, são também importantes e necessários.

\section{Fluxo de informações para familiares/acompanhantes}

No SHE I, o fluxo de informações para os familiares ocorre por meio de um Boletim Informativo, o qual é disponibilizado ao Serviço Social que, mediante manifestação de interesse por parte de familiares e amigos, informa sobre o estado de saúde dos pacientes a qualquer hora do dia. Essa conduta é favorável porque, um dos anseios de parentes próximos e amigos de pacientes que se encontram em SHE é o acesso rápido às informações atualizadas do estado de saúde do ente em tratamento ${ }^{(23)}$.

No SHE II, apesar de não existir Boletim Informativo, durante a semana, o médico disponibiliza um horário específico para informar/orientar individualmente os familiares dos usuários que se encontram na Sala de Emergência, caracterizados como de alta complexidade. Nos finais de semana e feriados, as orientações são fornecidas pelo médico plantonista. Contudo, os familiares dos pacientes internados em outros ambientes desse Serviço não recebem informações de maneira sistematizada.

Apesar de os fluxos de informações serem diferentes nos dois Serviços, se percebe que em geral os familiares/acompanhantes são informados sobre o estado de saúde do paciente. Essa é uma conduta importante e necessária, especialmente em SHE porque, na dinâmica do cuidado, a ocorrência de vínculo afetivo e social, entre o usuário e o familiar/acompanhante, tende a produzir resultados que favorecem a recuperação do paciente ${ }^{(24)}$.

Com relação às visitas, no SHE I existem dois horários ao dia. Já as informações, são fornecidas individualmente, pelo enfermeiro responsável pelo turno e/ou o médico plantonista. No SHE II, não foi observado horário específico para visitas e; as informações são fornecidas somente pelo médico plantonista, de acordo com a sua disponibilidade.

$\mathrm{Na}$ atualidade, as instituições de saúde têm se utilizado da Visita Aberta para criação de um ambiente favorável ao desenvolvimento da comunicação entre usuário/equipe/acompanhante ${ }^{(24)}$. Estratégias dessa natureza possibilitam a quebra de barreiras impostas pela hospitalização e tendem à promoção de um ambiente seguro e terapêutico ao paciente ${ }^{(25)}$.

Em que pese à importância da Visita Aberta, quando se trata de otimizar o tempo, esse recurso não se enquadra totalmente às necessidades dos SHE porque, nesses locais, a característica do atendimento é voltada às urgências e emergências as quais, em princípio, indicam que a permanência do paciente no Serviço deverá ser breve.

\section{Fluxo de usuários para o atendimento na recepção}

Essa subcategoria revelou a forma como ocorre a abordagem primária ao usuário e o seu encaminhamento a outras etapas do atendimento.

Um diferencial importante observado no SHE I é o fato de o usuário ser recepcionado por um colaborador (orientador de fluxo; recepcionista) no momento da sua chegada e também, após receber orientações sobre o atendimento. Ainda na Recepção, ele (o paciente) e/ou seu acompanhante é encaminhado para abertura da FAA e em seguida, a Consulta de Enfermagem. 
No SHE II, apesar de a Recepção ser o único referencial para o atendimento primário, após a abertura da FAA o usuário é direcionado ao local de espera, que se situa em frente do consultório da especialidade que o atenderá. Vale lembrar que, nos dois Serviços, a entrada e a primeira abordagem às vitimas graves acontecem em local distinto (Sala de Emergência) do atendimento geral ao público, o que é necessário, por se tratar de situações que demandam atendimento imediato.

Em alguns SHE, devido à alta demanda de usuários, o enfermeiro é o "primeiro rosto" que o paciente e os familiares vêem quando entram no hospital ${ }^{(6)}$. Nesse aspecto, o ACCR intervém decisivamente na humanização do atendimento e na reorganização do fluxo porque, de acordo com estudo publicado no ano de $2010^{(6)}$, o qual estuda as funções de um enfermeiro em sistemas de triagens, a formação acadêmica desse profissional proporciona a ele maiores subsídios para abordagens seguras e humanizadas ao usuário logo no momento da sua chegada ao serviço.

\section{CONCLUSÃO}

No contexto organizacional dos SHE, a definição correta de fluxos para o atendimento tende a resultar em ações sistematizadas e eficientes. Apesar de ações desse tipo, ser almejada por ambos os Serviços, se constatou que, na instituição que adota a diretriz $A C C R$, o fluxo de pacientes ocorre de forma contínua e hierarquizada; há responsabilização dos profissionais pelo atendimento e; o vínculo entre usuário e profissionais é mais próximo.

Em relação à prática do enfermeiro, observou-se que no SHE I, além de ocorrer maior valorização das atividades desenvolvidas, esse profissional tem maior autonomia na condução dos casos, pois ele é o principal responsável pela organização, gerenciamento e avaliação do atendimento inicial prestado ao paciente.

\section{REFERÊNCIAS}

1.Ministério da Saúde (BR). Secretaria Nacional de Organização e desenvolvimento dos serviços de saúde. Terminologia Básica em Saúde. Brasília (DF): Ministério da Saúde; 1987.

2. Souza MF, Figueiredo LA, Pinto IC. Análise da utilização do Serviço de Pronto-Socorro na Percepção do Usuário. CiencCuid Saúde. 2010;9(1):13-20.

3. Farrokhnia N, Göransson KE. Swedish emergency department triage and interventions for improved patient flows: a national update. Scand J Trauma ResuscEmerg Med. 2011; 19:72

4. Bittencour RJ, Hortale VA. Intervencões para solucionar a superlotação nos serviços de emergência hospitalar: uma revisão sistemática. CadSaude Publica $2009 ; 25(7): 1439-54$.

5. Stover-Baker B, Stahlman B, Pollack M. Triage nurse prediction of hospital admission. J EmergNurs. 2012; 38(3): 30610.

Rev. Eletr. Enf. [Internet]. 2015 jan./mar.;17(1):108-16. Disponível em: http://dx.doi.org/10.5216/ree.v17i1.23823. - doi: 10.5216/ree.v17i1.23823.
Outro achado importante do estudo é a constatação/confirmação de que nas instituições investigadas não se observou a existência de um sistema de contra-referência com a Rede Básica de Saúde, o que certamente resulta em maior congestionamento "de porta" nos SHE. Nesse sentido, estudos que abordam os mecanismos de referência e contra-referência na Rede de Serviços de Saúde são necessários e urgentes, visto que a inexistência ou mesmo o funcionamento inadequado desses mecanismos comprometem a segurança do paciente.

Conclui-se que o fluxo de atendimento em SHE que atua com o ACCR é mais dinâmico, sistematizado e seguro porque, além da priorização dos casos graves, as anotações de enfermagem, a sequência interna para a realização de exames complementares, a administração de medicamentos e às reavaliações clínicas são também direcionadas de acordo com a gravidade de cada caso.

Como limitação deste estudo considera-se o fato de os seus resultados serem fruto das observações e interpretações dos seus pesquisadores e não dos trabalhadores que atuam nos Serviços e que poderiam fornecer testemunhos com mais objetividade.

Às questões que envolvem o exercício da observação do fluxo do atendimento representam um campo fértil para estudos que visem melhorias nos SHE. Nesse sentido, sugerem-se investigações com enfoque mais amplo, tais como aquelas voltadas ao estudo das relações internas e externas do fluxo de atendimento e; identificação das potencialidades e fragilidades do ACCR em SHE, considerando as características de cada instituição. No campo da prática, aos gestores, trabalhadores e, especialmente enfermeiros que atuam em SHE e que atendem os usuários por ordem de chegada, sugere-se a implantação e o monitoramento da diretriz ACCR.

6. Montejano AC, Visser LS. What is a Triage Nurse? J Emerg Nurs. 2010; 36 (1): 85-86.

7. Grupo Brasileiro de Acolhimento com Classificação de Risco. História da Classificação de Risco. [Internet]. [citado 2013 abr 04]. [cerca de 3 p.]. Available from:

http://www.gbacr.com.br/index.php?option=com_content\&task= blogsection\&id $=8 \&$ Itemid $=39$.

8. Buschhorn HM, Strout TD, Sholl JM, Baumann MR. Emergency Medical Services Triage using the Emergency Severity Index: is it reliable and valid? Journal of Emergency Nursing. 2013; 5(39):55-63.

9. Bullard MJ, Hunger B, Spense J, Grafistein E. Revisions to the Canadian Emergency Department Triage and Acuity Scale (CTAS) adult guidelines. CJEM - JCMU. 2008; 10 (2): 136-42.

10. Janssen M, Von Achterberg T, Adriaansen M, Kampshoff C, Mintjes-de Groot J. Adherence to the guideline "Triage in 
emergency departments": a survey of Dutch emergency departments. J ClinNurs. 2011; 20 (17-18): 2458-68.

11. Ministério da Saúde (BR). Secretaria Executiva. Núcleo Técnico da Política Nacional de Humanização (HumanizaSus). Acolhimento com avaliação e classificação de risco: um paradigma ético-estético no fazer em saúde. Brasília (DF): Ministério da Saúde; 2004.

12. Ministério da Saúde (BR). Secretaria de Atenção à Saúde. Acolhimento e Classificação de Risco nos serviços de Urgência. Brasília (DF): Ministério da Saúde; 2009.

13. Desmettre T, Baron AF, Capellier G, Tazarourte K.

L'infirmièreorganisatrice de l'accueil (IOA) :rôle et fonctions. Réanimation . 2013; 22(6):610 -615.

14. Polit DF, Beck CT. Fundamentos de Pesquisa em enfermagem - Avaliação de evidências para a prática da enfermagem. 7a Ed. Porto Alegre: Artmed; 2011. P. 669

15. Linden CVD, Lindeboom R, Linden NVD, Lucas c. Managing patient flow with triage streaming to identify patients for Dutch emergency nurse practitioners. IntEmergNurs. 2012; 20(2): 5257.

16. Perondi MBM, Sakano TMS, Schvartsman C. Utilização de um sistema informatizado de atendimento em pronto-socorro pediátrico com sistema de escore clínico de triagem. einstein. 2008;6(1):31-6.

17. Silva AP, Diniz AS, Araújo FA, Souza CC. Presença da queixa de dor em pacientes classificados segundo o protocolo de Manchester. R. Enferm. Cent. O. Min. 2013;3(1):507-517 18. Soremekun AO, Capp R, Biddinger PD, White BA, Chang $Y$, Crignan SB, Brown DF. Impact of physician screening in the emergency department on patient flow. J Emerg Med. 2012. 43(3): 509-15.

19. Bertrand SW. Registred Nurses Integrate Traditional Chinese Medicine Into the Triage Process. Qual Health Res. 2012; 22(2): 263-73.

20. Shea SS, Hoyt KS. "Rapid" Team Triage: One Hospital's Approach to Patient-Centered Tem Triage. AdvEmergNurs. 2012; 34(2): 177-89.

21. Mendes EV. As redes de atenção à saúde. Brasília: Organização Pan-Americana da Saúde, 2011.

22. Oliveira MLF, Scochi MJ. Determinantes da utilização dos serviços de urgência/emergência em Maringá (PR). CiencCuid Saúde. 2002;1(1):123-28.

23. Marques GQ, Lima MADS. Demandas de usuários a um serviço de Pronto Atendimento e seu acolhimento ao sistema de saúde. Rev Latino-Am Enfermagem. [Internet]. 2007 [Cited 2010 jan 01];15(1): [Cerca de 8 p.]. Available from: http://www.scielo.br/pdf/rlae/v15n1/pt_v15n1a03.pdf. 24. Sanches ICP, Couto IRR, Abrahao AL, Andrade M. Acompanhamento hospitalar: direito ou concessão ao usuário hospitalizado? Ciênc. saúde coletiva. 2013, 18(1):67-76. 25. Hayden $C$, Burlingame $P$, Thompson $H$, Sabol VK. Improving Patient Flow in the Emergency Department by Placing a Family Nurse Practitioner in Triage: A Quality-Improvement Project.J EmergNurs. 2014; 40(4):346-51.

Artigo recebido em 13/05/2013.

Aprovado para publicação em 10/06/2014.

Artigo publicado em 31/03/2015. 